\title{
"Magnetoscan": A Modified Hall Probe Scanning Technique for the Detection of Inhomogeneities in Bulk High Temperature Superconductors
}

\author{
M. Eisterer, S. Haindl, T. Wojcik and H. W. Weber \\ Atominstitut der Österreichischen Universitäten, A-1020 Vienna, Austria \\ E-mail: eisterer@ati.ac.at
}

\begin{abstract}
We present a novel technique for the investigation of local variations of the critical current density in large bulk superconductors. In contrast to the usual Hall probe scanning technique, the sample is not magnetized as a whole before the scan, but locally by a small permanent magnet, which is fixed near the Hall probe, during the scanning process. The resulting signal can be interpreted as a qualitative measure of the local shielding currents flowing at the surface.

PACS numbers: 74.81.Bd, 74.25.Qt, 06.60.Mr
\end{abstract}

\section{Introduction}

Melt textured bulk superconductors are promising candidates for technical applications such as permanent magnets, motors or flywheels. These applications require macroscopically homogeneous properties in the largest possible specimens. However, melt processed materials are strongly inhomogeneous, at least on a microscopic scale 1, since the formation of defects, e.g. 211 particles, microcracks, twin planes, subgrain boundaries, stacking faults, dislocations, oxygen deficient regions or the boundaries between the growth sectors, cannot be avoided during the growth process. These defects influence the transport properties in various ways. Large normal conducting inclusions simply reduce the superconducting cross section, cracks impede the current flow locally, (sub)grain boundaries reduce the critical current density across them [2], but some defects can also act as pinning centers and, therefore, improve the transport properties. The defect structure is related to the growth conditions [3, 4]. Differences were found between the a-growth and the c-growth sectors [5] and the defect concentration generally increases with increasing distance from the seed.

The experimental characterization of the transport properties in large samples is commonly made by scanning the remnant flux profile. This technique is rather insensitive to material inhomogeneities 6, since all parts of the sample volume contribute to the magnetic field at each measured point. Even if the current distribution can be estimated under certain assumptions [7, it is generally impossible to assess the correlation between the critical currents and the local microstructure, since $J_{c}$ does not only depend on the microstructure, but also on the magnetic field, which changes within the sample. Therefore, a new technique - the "magnetoscan" - has been developed. Here, the currents are induced only locally over a small sample area, where the magnetic field remains nearly constant. Moving the magnet enables us to compare the local transport properties at different positions of the sample with a spatial resolution of less than one 


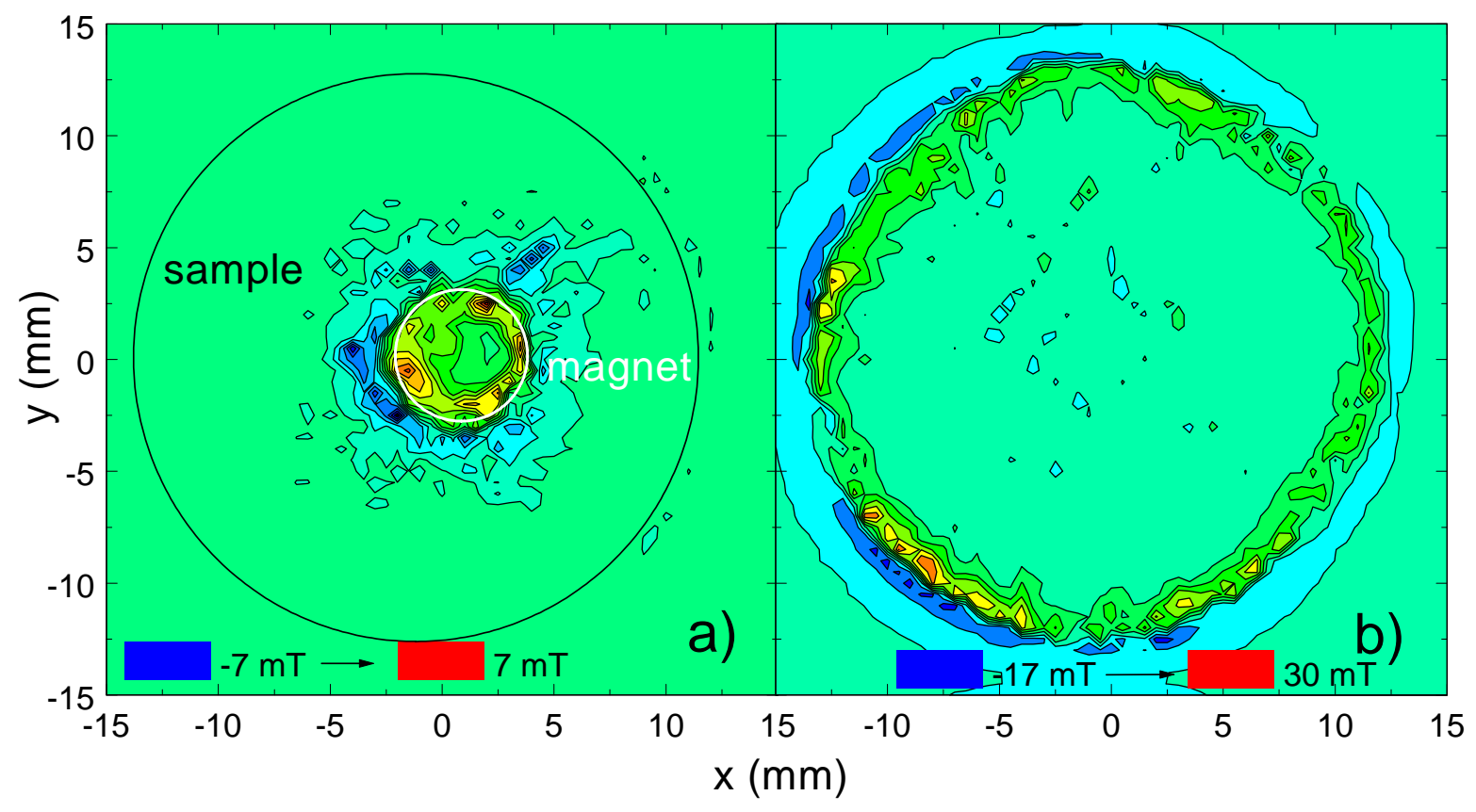

Figure 1. (a) Trapped flux profile after touching the surface of the superconductor with a permanent magnet. The white and black circles indicate the position of the magnet and sample, respectively. (b) Remnant flux after a magnetoscan recovered by conventional field mapping.

millimeter. The magnetoscan, therefore, represents an ideal tool to relate the local $J_{c}$ to microstructural investigations, in order to optimize the growth process in the end.

\section{The principle: Local shielding currents induced by a permanent magnet}

Although the details of the response of a superconductor to a nonuniform magnetic field induced by a permanent magnet are quite complex 8 , shielding can be understood qualitatively, if the permanent magnet is much smaller than the superconductor and if the magnet is placed close to the surface, but not close to the edge of the superconductor.

The following experiment was made. A cylindrically shaped permanent magnet was moved vertically to the surface of a large bulk sample $(R=12.9 \mathrm{~mm}, h=10.8 \mathrm{~mm})$, which had been zero field cooled in liquid nitrogen. The magnet with a radius $R=3 \mathrm{~mm}$ and a height $h=18 \mathrm{~mm}$ had a magnetic induction of $100 \mathrm{mT}$ at the center of its top surface. After removing the magnet, the remnant flux profile of the sample was scanned by a conventional Hall probe scanner at a distance of $0.2 \mathrm{~mm}$ above the surface (Fig. [1). Flux penetrated the sample near the edge of the magnet (white circle), whereas no flux is trapped in the center (the inner profile is built up by removing the magnet). Flux penetrates and leaves (bright spots) the sample very inhomogeneously, which must be related to the local properties of the sample.

This observation represents the basis for the method presented in this paper. Flux penetrates more easily in regions with "bad" transport properties, the response of the sample is smaller there. In regions with high shielding currents, the feedback field of the sample is larger. The flux penetration at the edge of the sample (black circle in Fig. Ta) is not important for our purpose and results from the large demagnetization there. If the magnet is moved horizontally over the sample surface, the flux profile could either move simultaneously or flux could (partly) be trapped in the superconductor. This can 


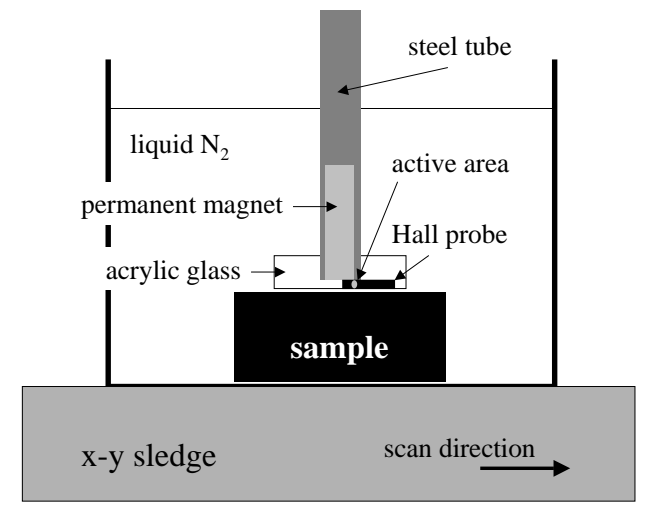

Figure 2. Modified Hall probe scanning device

be checked by mapping the remnant flux after moving the magnet parallel to the sample surface along the typical path of a Hall scan (Fig. 1b). We find that flux is trapped at the edge of the sample, but (almost) not inside. This indicates that hysteretic memory effects due to the scanning process are absent, apart from the sample edge.

The penetration depth of the magnetic field is roughly estimated from the relation $\delta=B / \mu_{0} J$. With a typical critcal current density of $10^{8} \mathrm{Am}^{-2}$, the penetration depth of the field of this permanent magnet is expected to be slightly smaller than $1 \mathrm{~mm}$.

\section{Magnetoscan: Experimental Setup and Tests}

Fig. 2 2 shows the experimental setup schematically. A Hall probe (AREPOC HHPVPO) with an active area of $50 \times 50 \mu \mathrm{m}^{2}$ is fixed on the edge of the small permanent magnet used above. The permanent magnet and the Hall probe remain fixed, while the sample is moved together with the dewar by a $\mathrm{x}-\mathrm{y}$ sledge. The gap between the active area of the Hall probe and the sample surface is about $0.2 \mathrm{~mm}$, between the permanent magnet and the sample about $1 \mathrm{~mm}$. Measurements of the Hall voltage were taken on a 0.25 or $0.5 \mathrm{~mm}$ grid. A preliminary scan was made with the Hall probe positioned at the center of the magnet's surface, not at the edge. In this case the signal nearly did not change apart from the sample edge. This behavior is expected from the above considerations about the magnet's "fingerprint", since the magnetic field was found to be zero at the center of the magnet. Only the transition from nearly perfect screening above the sample to no screening beside it is observed.

Figure 3 a shows a typical example of a magnetoscan (with the Hall probe at the edge of the magnet). The arrow indicates the direction of the relative movement of the magnet and the Hall probe. The Hall probe always follows the magnet (when measurements are taken) in order to minimize the remaining hysteretic effects. The change of the signal is much faster, when the Hall probe reaches the sample, since the magnet is already above the sample in this case, while on the opposite edge, the magnet has already left the sample. Due to the rapidly changing geometrical conditions, the signal cannot be evaluated in terms of current transport properties there. A further magnetoscan was made after rotating the sample by $180^{\circ}$. As expected, the geometrical effects near the sample edge remained unchanged, whereas the details of the magnetoscan caused by inhomogeneities were also rotated by $180^{\circ}$.

It should be mentioned that the signal is very sensitive to the gap between the Hall probe and the sample surface. Only samples with a flat surface can be investigated. If the (flat) sample suface is not oriented completely horizontally, the signal increases 
with decreasing gap, but the inhomogeneities are still detectable, because the systematic influence of such a misalignment and of local changes caused by inhomogeneities can be easily distinguished.

\section{Results}

Figure $3 \mathrm{~b}$ shows the details of the magnetoscan discussed above (Fig 3 a) just for the inner part of the sample. The difference in the Hall voltage between two contour lines is now smaller by a factor of 7 compared to Fig. 3a. The usual trapped flux profile (0.2 $\mathrm{mm}$ above the sample surface) is plotted for comparison in Fig. 3. $\left(B_{T}^{\max }=0.54 T\right)$. Three interesting features can be observed in this magnetoscan: the irregularly shaped contour lines, the smaller response at the position, where the seed was located, and the highest values near the boundaries between the different a-growth sectors (white lines in Fig. [3b).

In order to confirm the relation of these findings to the local transport properties, the conventional trapped field profile was measured $0.2 \mathrm{~mm}$ and $0.4 \mathrm{~mm}$ above the sample surface. The difference of these two profiles should be approximately proportional to the derivative of $B_{z}$ with respect to the vertical coordinate, i.e. $\partial B_{z} / \partial z$. This quantity is strongly influenced by the surface currents as follows from the BiotSavart law:

$$
\mathbf{B}(\mathbf{r})=\frac{\mu_{0}}{4 \pi} \int_{V} \frac{\mathbf{J}\left(\mathbf{r}^{\prime}\right) \times\left(\mathbf{r}-\mathbf{r}^{\prime}\right)}{\left|\mathbf{r}-\mathbf{r}^{\prime}\right|^{3}} d^{3} r^{\prime}
$$

The integrand does not depend on $z$ alone, but always on $z-z^{\prime}$. Therefore, in the integral for $\mathbf{B}\left(\mathbf{r}+\delta \mathbf{e}_{z}\right)$ (in the difference quotient) $\mathbf{r}^{\prime}$ can be substituted by $\mathbf{r}^{\prime}-\delta \mathbf{e}_{z}$, and, if $\partial \mathbf{J} / \partial z$ exists, the partial derivative of $\mathbf{B}$ with respect to $z$ can be expressed by

$$
\begin{aligned}
\frac{\partial \mathbf{B}}{\partial z}(\mathbf{r})= & \left.\frac{\mu_{0}}{4 \pi} \int_{F} \frac{\mathbf{J}\left(\mathbf{r}^{\prime}\right) \times\left(\mathbf{r}-\mathbf{r}^{\prime}\right)}{\left|\mathbf{r}-\mathbf{r}^{\prime}\right|^{3}}\right|_{z^{\prime}=-h / 2} d x^{\prime} d y^{\prime} \\
& -\left.\frac{\mu_{0}}{4 \pi} \int_{F} \frac{\mathbf{J}\left(\mathbf{r}^{\prime}\right) \times\left(\mathbf{r}-\mathbf{r}^{\prime}\right)}{\left|\mathbf{r}-\mathbf{r}^{\prime}\right|^{3}}\right|_{z^{\prime}=h / 2} d x^{\prime} d y^{\prime} \\
& +\left.\frac{\mu_{0}}{4 \pi} \int_{V} \frac{\partial \mathbf{J}}{\partial z}\right|_{\mathbf{r}=\mathbf{r}^{\prime}} \times \frac{\left(\mathbf{r}-\mathbf{r}^{\prime}\right)}{\left|\mathbf{r}-\mathbf{r}^{\prime}\right|^{3}} d^{3} r^{\prime}
\end{aligned}
$$

The first and the second term are integrations over the bottom $\left(z^{\prime}=-h / 2\right)$ and top $\left(z^{\prime}=h / 2\right)$ surface of the sample, respectively. If the field profiles are measured close to the top surface, the first term can be neglected. The influence of the third term is a priori unknown. If the variation of the currents along the sample thickness is smooth (at least near the top surface), which ensures the existence of $\partial \mathbf{J} / \partial z$, its contribution is small, otherwise it could become dominant. If it is negligible, the difference of two field profiles measured at slightly different heights (Fig. 3 d), is proporional to the field profile of the surface currents (second term). The qualitative agreement with the magnetoscan is satisfactory. Note that the two profiles are not expected to be identical, since currents all over the surface contribute to the differential profile (plus a contribution of the whole sample), while the currents are induced only locally during the magnetoscan. For instance, a completely homogeneous current density results in a constant signal during the magnetoscan (apart from the edges), but the corresponding differential profile has a sharp peak at the center of the sample (field of a thin sample). Nevertheless inhomogeneities can be detected in both cases. The regions with weak 


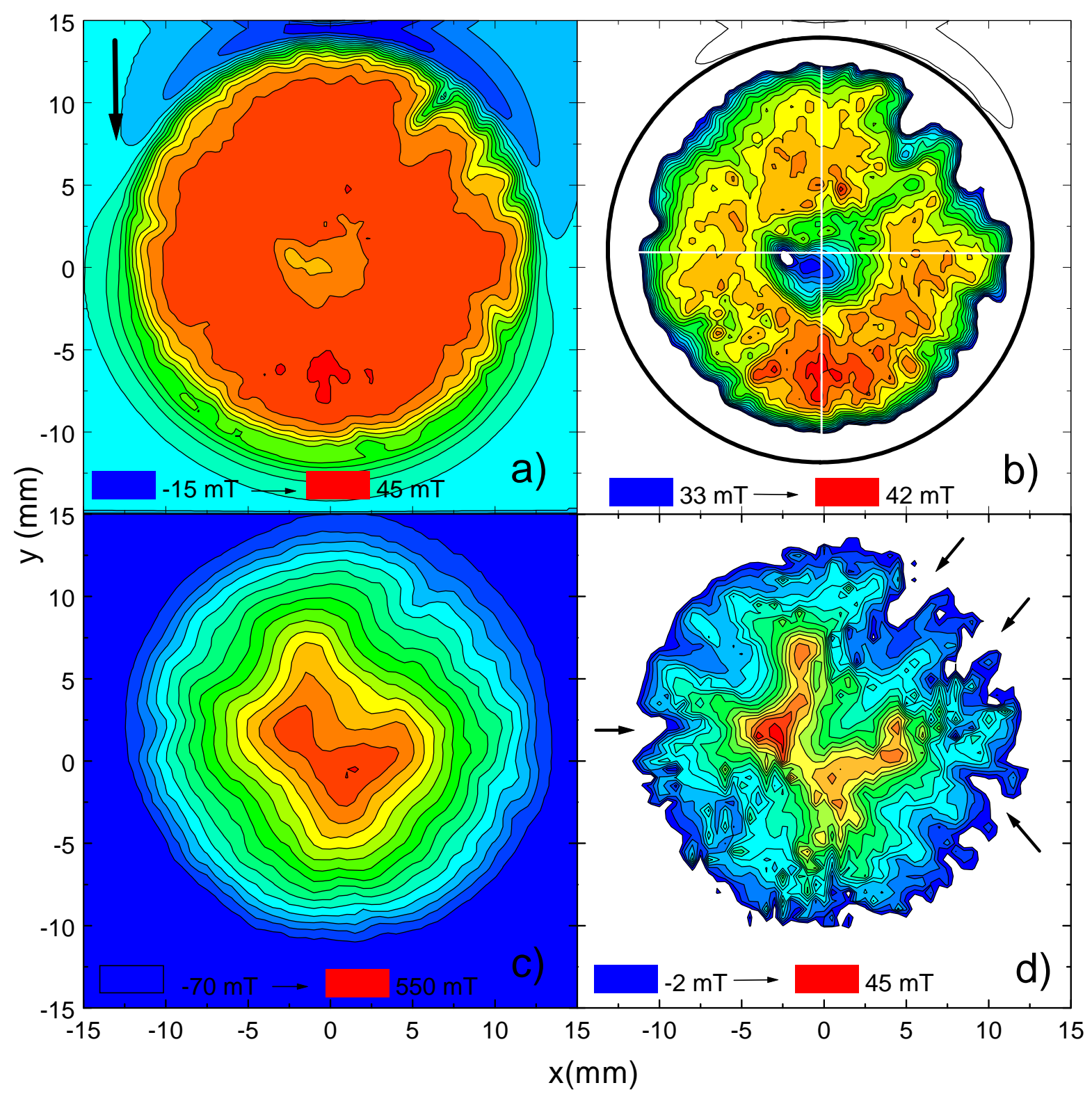

Figure 3. (a,b) Example of a magnetoscan, plotted on different scales. The solid circle indicates the circumference of the sample, the white lines indicate the boundaries of the growth sectors. (c) Usual trapped flux profile of the same sample. (d) Difference betweem the trapped flux profiles measured at $0.2 \mathrm{~mm}$ and $0.4 \mathrm{~mm}$ above the sample surface.

currents near the sample edge (indicated by arrows in Fig. 31), the higher currents at the boundaries of the a-growth sectors and the reduction of the currents directly below the seed are clearly observed with both methods.

Further examples of magnetoscans are plotted in Fig. 4. The dimension of the sample was $R=13.1 \mathrm{~mm}$ and $h=9.8 \mathrm{~mm}$, its maximum trapped field after dc activation was $0.79 \mathrm{~T}$. The profile of the top side (a) is nearly quadratic, although the sample is cylindrically shaped. The best shielding is again observed near the growth boundaries (oriented horizontally and vertically) and a depression of the shielding currents is found at the former position of the seed. The weaker shielding near the seed (indicated by the white arrow) corresponds to a bright spot, which is visible at the sample surface. At the 


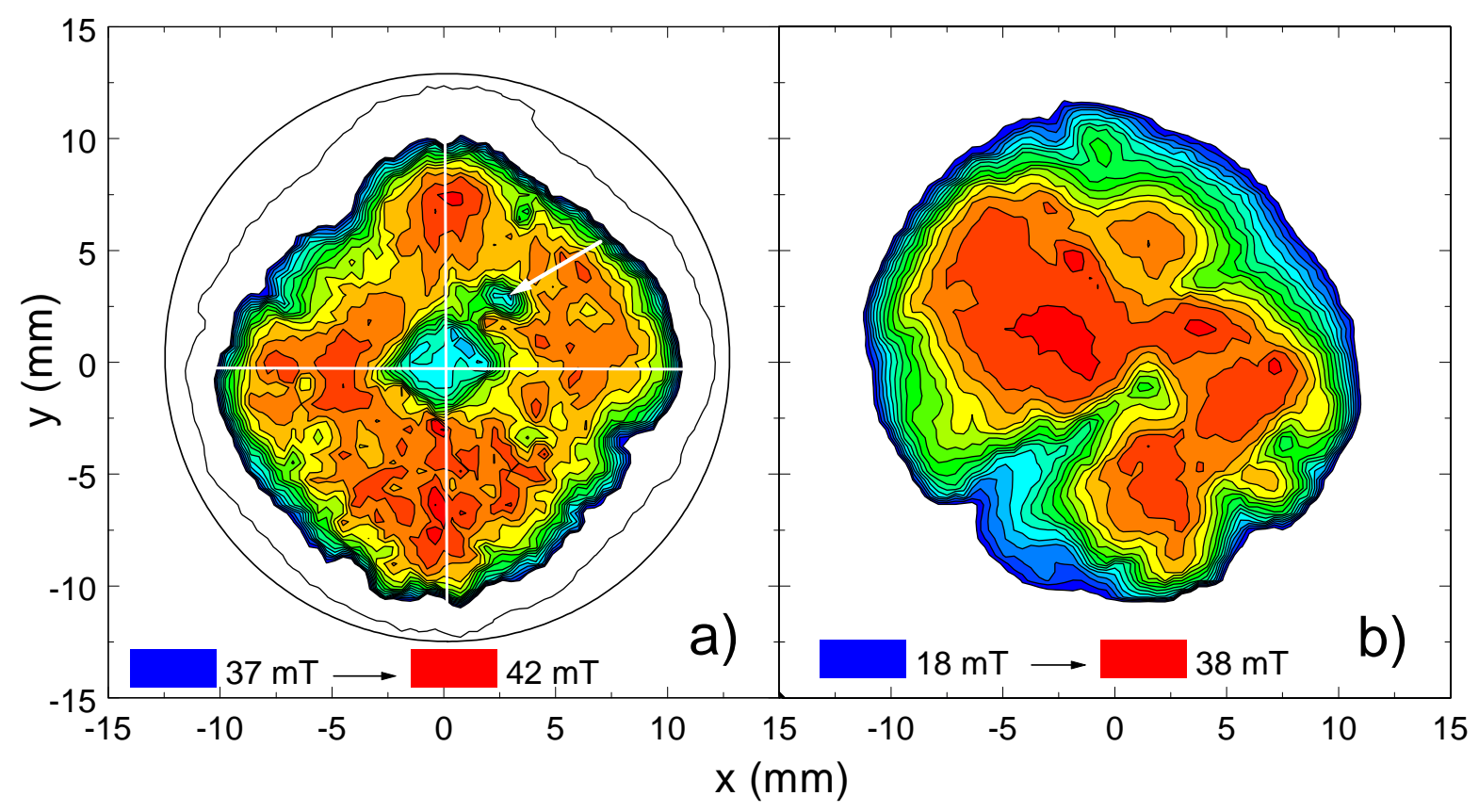

Figure 4. Magnetoscan (a) of the top side, (b) of the bottom side of the sample.

bottom side (Fig. 4b) the transport properties are inhomogeneous on a larger scale. By optical inspection of the sample, two types of differently looking regions are observed at the bottom side, i.e. crystalline regions with a pronounced subgrain structure and rather dull black looking areas. The shape of these areas is nicely displayed by the magnetoscan with higher shielding currents in the crystalline regions.

Although many inhomogeneities can be ascribed to optically visible features of the samples (position of the seed, growth sector boundaries etc.), there remain peculiarities in the results, which cannot be correlated with visible inhomogeneities, e.g. the reasons for the irregular (cf. 3b) or quadratic (cf. 4a) shape of the magnetoscan profiles.

\section{Conclusions}

Magnetoscanning represents a simple method to investigate changes of the transport properties of large bulk superconductors with a spatial resolution of around one millimeter. In contrast to the usual scanning Hall probe method, shielding currents are induced only locally. The range of magnetic field penetration can be chosen by the size and the strength of the permanent magnet or could even be varied by using an electromagnet.

\section{References}

[1] Diko P, Gawalek W, Habisreuther T, Klupsch T and Görnert P 1995 Phys. Rev. B 5213658

[2] Sandiumenge F, Vilalta N, Rabier J and Obradors X 2001 it Phys. Rev. B 64184515

[3] Salama K, Selvamanickam V, Gao L and Sun K 1989 Appl. Phys. Lett. 542352

[4] Ogava N, Hirabayashi I and Tanaka S Physica C $\mathbf{1 7 7} 101$

[5] Diko P, Zmorayova K, Granados X, Sandiumenge F and Obradors X 2003 Physica C 384125

[6] Gonzalez-Arrabal R, Eisterer M and Weber H W 2003 J. Appl. Phys. 934734

[7] Carrera M, Amorós J, Carrillo A E, Obradors X and Fontcuberta J 2003 Physica C 385539

[8] Qin M J, Li G, Liu H K, Dou S X and Brandt E H 2002 Phys. Rev. B 66024516 